\title{
Etude Sedimentologique Et Stratigraphique Du Remplissage Du Premier Etage Du Gisement De Montgaudier, Charente, France
}

\author{
Boukhir Mohammed, (Associate Professor) \\ Chaouni Abdel-Ali, (Professor) \\ Lahrach Abderrahim, (Professor) \\ Laboratoire Géoressources et Environnement \\ Université Sidi Mohamed Ben Abdellah, Maroc
}

doi: 10.19044/esj.2017.v13n21p183 URL:http://dx.doi.org/10.19044/esj.2017.v13n21p183

\begin{abstract}
The study of the deposits accumulated over time in the first stage of the Montgaudier site, at the level of the two sectors, confirmed that these sediments were non-native and were put in place by runoff. Concretion processes have transformed the sandy sediments from the western part of this filling into a sandy bank. The detailed granulometric analyzes of these deposits have made it possible to establish the palaeoclimatic factors, rather temperate and humid, which presided over them. These factors were defined by the shiny bluntness of the quartz grains of these sediments. Finally, the sediment stratigraphy of the two sectors and their correlation with the deposits of the Gaudry shelter allowed the establishment of a relative chronology of this filling.
\end{abstract}

Keywords: Sedimentology, Stratigraphy, Deposit, First Floor, Montgaudier, Charente, France

\section{Résumé}

L'étude des dépôts qui se sont accumulés avec le temps à l'intérieur du premier étage du site de Montgaudier, au niveau des deux secteurs, ont permis de confirmer que ces sédiments sont allochtones et ont été mis en place par ruissellement. Des processus de concrétionnement ont transformé les sédiments sableux de la partie ouest de ce remplissage en un banc gréseux. Les analyses granulométriques détaillées de ces dépôts ont permis d'établir les facteurs paléoclimatiques plutôt tempéré et humide qui présidé leur mis en place. Ces facteurs ont été précisés par l'aspect émoussés luisants des grains de quartz de ces sédiments. Enfin, la stratigraphie des sédiments 
des deux secteurs et leur corrélation avec les dépôts de l'abri Gaudry ont permis l'établissement d'une chronologique relative de ce remplissage.

Mots-clés : Sédimentologie, Stratigraphie, Gisement, Premier étage, Montgaudier, Charente, France

\section{Introduction}

Le gisement de Montgaudier est l'un des plus importants sites paléolithiques de Charente. Le remplissage de cette grotte de Montgaudier se présente de façon très complexe. Il a fait l'objet d'une étude sédimentologique, stratigraphique et minéralogique. Son étude sédimentologique demeure du plus haut intérêt, afin de mieux connaitre sa lithostratigraphie globale et d'essayer de le situer dans le cadre chronologique du Quaternaire charentais.

Le présent travail comportera les résultats de recherches effectuées sur la partie premier étage ou locus 6.

\section{Situation géographique du gisement de Montgaudier (fig.1).}

Le gisement de Montgaudier se situe à $2 \mathrm{~km}$ au Nord-ouest de la commune de Montbron à une vingtaine de kilomètres de la ville d'Angoulème, département de Charente. De coordonnées géographiques $45^{\circ} 41^{\prime}$ latitude Nord, $0^{\circ} 28^{\prime}$ longitude Est, Cette grotte s'ouvre dans les calcaires dolomitiques bajociens à une altitude de près de $100 \mathrm{~m}$ altitude. Il se localise sur la rive droite de la Tardoire, affluent de la Charente, France. Elle est composée de plusieurs loci et abris plus ou moins conservés dont les sédiments de remplissage montent une stratigraphie très complexe (Boukhir, 1992 et 2017 ; Debenath, 1974).

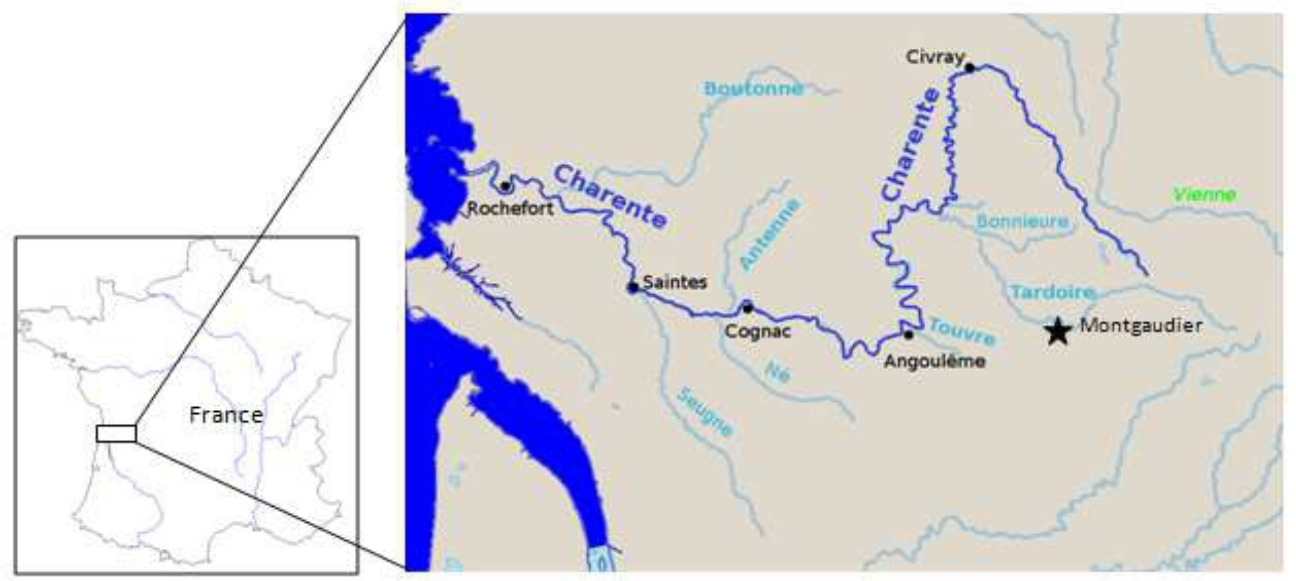

Fig.1 Situation géographique du gisement de Montgaudier 


\section{Situation des secteurs et profils étudiés au premier étage (fig. 2 et 3)}

Le premier étage du Gisement du Montgaudier est subdivisé en deux secteurs à savoir, les secteurs I et II.

- Secteur I, également subdivisé en deux profils 1 et 2.

- Le premier profil 1 (ou zone A) est orienté vers le Nord-est.

- Le second profil 2 est orienté vers le Nord et est séparé en deux zones : Zones B et C.

Trois séries d'échantillons ont été réalisées dans ce secteur : une première série au niveau de la zone $\mathrm{A}$, et deux autres séries au niveau des zones $\mathrm{B}$ et $\mathrm{C}$.

- Secteur II, se situe à l'Ouest du secteur I. La coupe établie dans ce secteur s'étend sur plus de $11 \mathrm{~m}$ de long. Deux séries d'échantillons ont été prélevées également : Séries 1 et 2

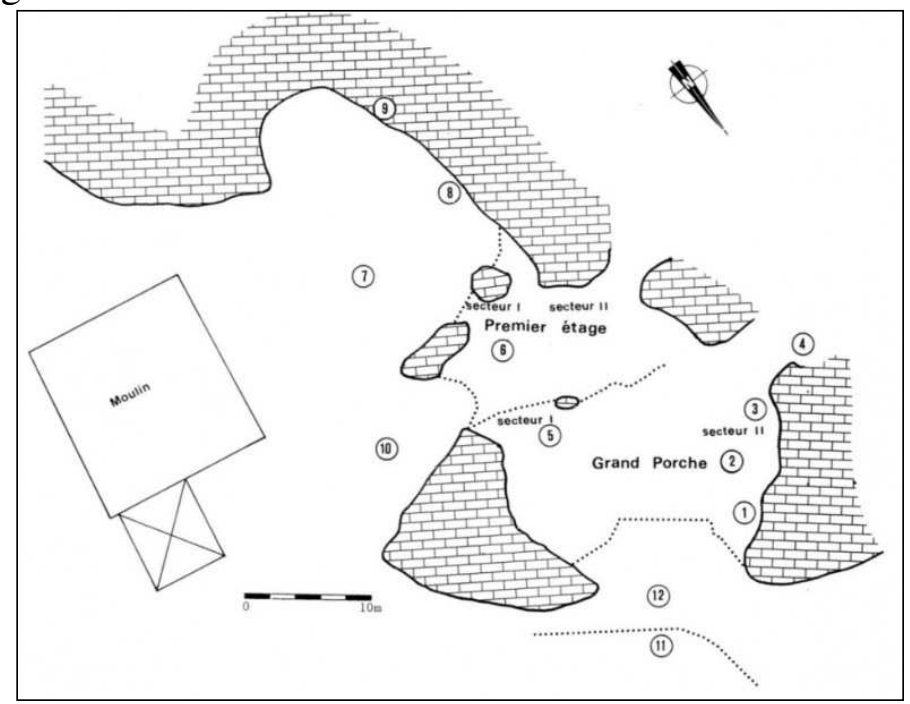

Fig. 2 : Plan général de Montgaudier : situation des secteurs I et II au premier étage.

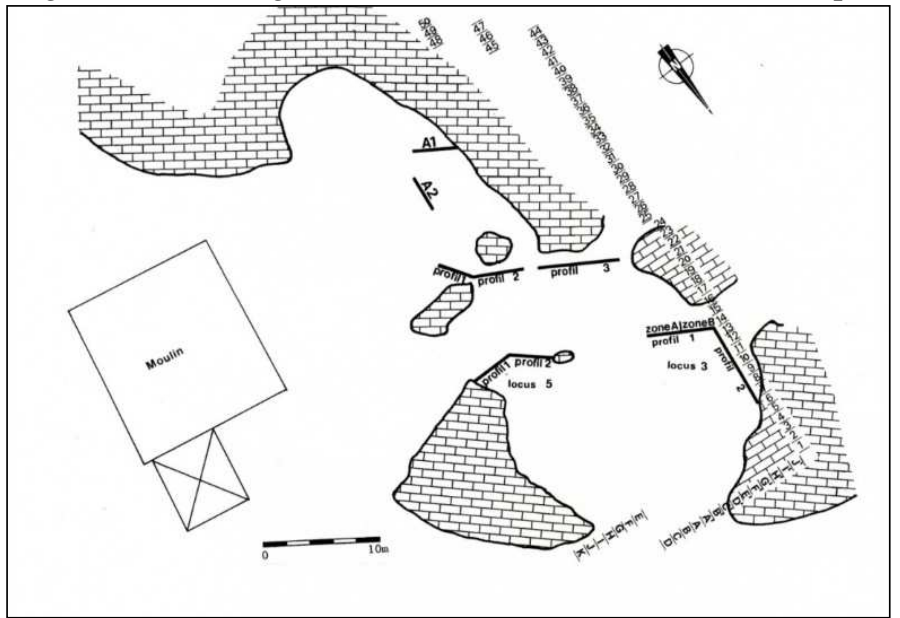

Fig. 3 : Localisation des différents profils réalisés au Grand porche 


\section{Stratigraphie}

\section{Secteur I : profil 1 (zones A) (Fig.4)}

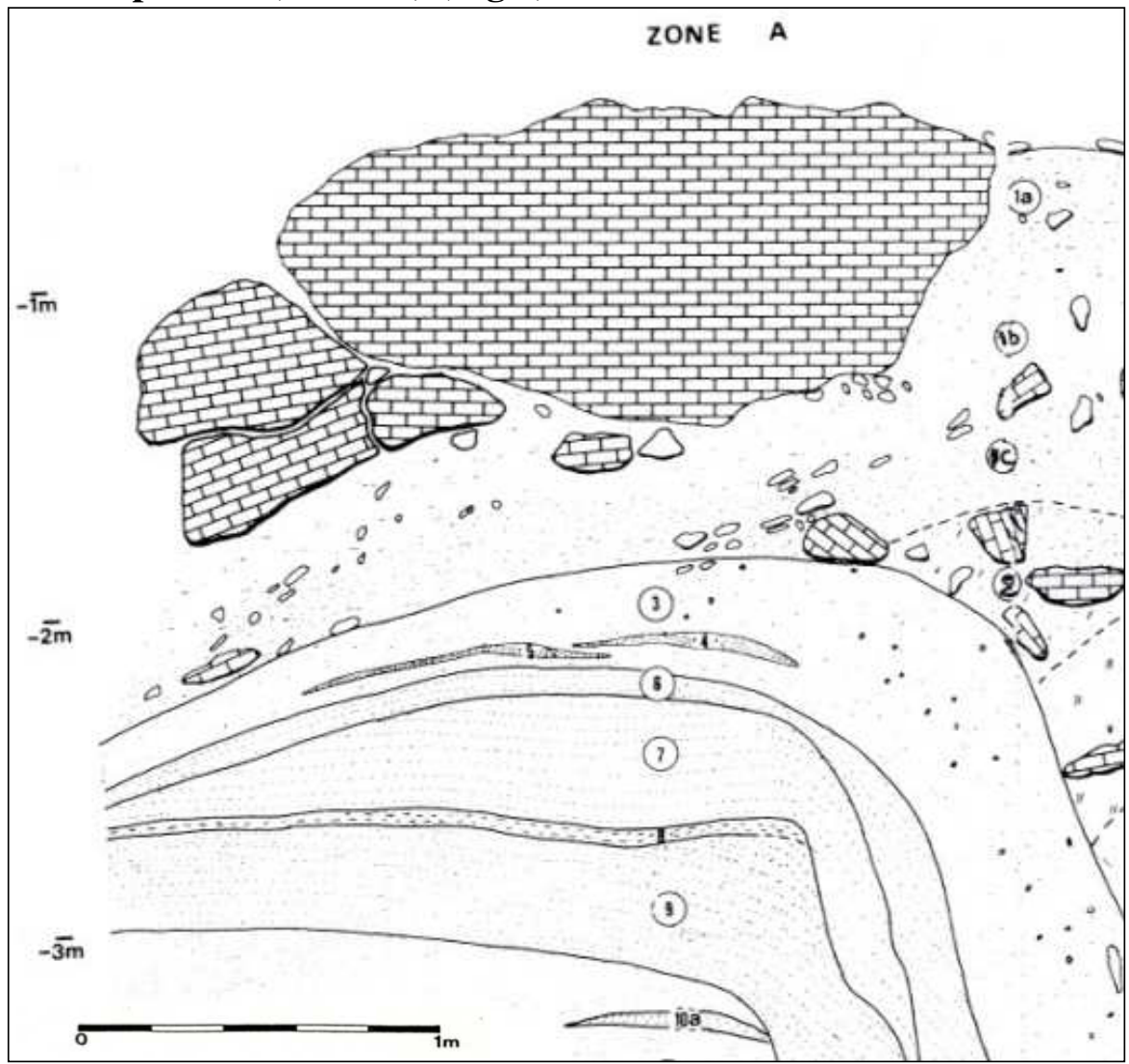

Fig. 4 : Coupe stratigraphique du profil 1, secteur I, zone A.

On distingue dans cette zone deux types de dépôts appartenant à deux ensembles différents :

Un ensemble inférieur: regroupant les couches 10 à 3, dont les dépôts sont en place géologiquement et archéologiquement.

Un ensemble supérieur : formé par les dépôts de la couche 2 et 1 qui ont glissé sur les couches sous-jacentes par éboulement et qui n'est donc pas archéologiquement en place. Du point de vue stratigraphique, on distingue de bas en haut les unités stratigraphiques suivantes :

Couche 10 : elle est visible sur une épaisseur de 1 mètre au maximum. Sa base n'a pas été atteinte par les fouilles. Il s'agit d'un sédiment fin de texture limono-sablo-argileuse. Ce dépôt présente une structure laminaire. Il est assez compact et de couleur brun très pale à l'état sec. La limite inférieure de cette couche est nette et régulière. Au sein de ce dépôt sont intercalés des sédiments sableux sous forme de lentilles, que nous avons séparés en deux niveaux distincts : Niveau 10a: formé par une lentille 
d'épaisseur maximale de $10 \mathrm{~cm}$ et de longueur $60 \mathrm{~cm}$ environ. A l'état sec, le sédiment est un sable de couleur jaune, non structuré et peu compact. Les limites de cette lentille sont brutales. Niveau $10 \mathrm{~b}$ : il est constitué par deux petites lentilles moins importantes que celle du niveau 10a. Le sédiment a une texture sableuse. Il est de couleur brun jaunâtre. Les limites de ces deux lentilles sont nettes.

Couche 9: d'épaisseur maximale $50 \mathrm{~cm}$, elle plonge vers le Nordouest et présente un pendage de $80^{\circ}$. Cette couche s'amenuise progressivement en allant vers le Sud-est du profil pour ne s'observer que sur $30 \mathrm{~cm}$ d'épaisseur. Le sédiment est composé de sables micacés et présente une structure litée. Il est assez compact à l'état sec. On trouve également des traces d'activités biologiques. A l'intérieur de cette couche se dessine une poche de sels manganiques à contours diffus.

Couche 8 : C'est une mince couche d'épaisseur maximale $5 \mathrm{~cm}$. Elle est limitée dans une grande partie sud-est de ce profil. Cependant, elle parait être érodée vers le nord-ouest de la coupe. Le sédiment est de texture très argileuse faiblement sablo-limoneuse, de structure laminaire. Il est bioturbé localement par des terriers sans toutefois perdre son aspect lité. Le dépôt est assez compact, et de couleur jaune. Sa limite inférieure est brutale et régulière.

Couche 7 : d'épaisseur maximale $40 \mathrm{~cm}$, elle plonge également vers le nord-ouest et recouvre de ce fait une partie de la couche 9. Dans cette même partie du profil, elle s'amincit rapidement pour disparaitre en biseau. Le sédiment de cette couche est un sable à micas blanc avec de minces passages limoneux. Le dépôt est peu compact, de structure laminaire peu développée, et de couleur brun très pâle. On note également la présence de traces d'oxydes de manganèse au sein de ces sédiments.

Couche 6 : elle surmonte la couche 7 sur une épaisseur qui varie de 10 à $20 \mathrm{~cm}$. Elle est fortement inclinée au nord-ouest où elle entre en contact avec la couche 9 avant de disparaitre sous la couche 3. Vers le sud-est de la coupe, elle a également tendance à s'incliner de façon progressive tout en perdant légèrement de son importance sans toutefois disparaitre définitivement. Le dépôt est composé essentiellement de sables à muscovite.

Couche 5: elle apparait sous forme d'une lentille d'épaisseur maximale $5 \mathrm{~cm}$ et de longueur 1 mètre environ. Elle est disposée horizontalement au sein des dépôts de la couche 3. Le sédiment est de texture sablo-limono-argileuse et peu compact. La limite de cette couche est nette et assez régulière. Le sédiment présente également des traces d'oxyde de manganèse.

Couche 4 : comme la couche 5, cette couche se développe sous forme de lentille horizontale au milieu de la couche 3 et elle a une épaisseur 
maximale de $6 \mathrm{~cm}$. Sa texture est comparable à celle de la couche 5 . Cependant, elle en diffère par une couleur jaune.

Couche 3: d'épaisseur maximale $40 \mathrm{~cm}$, cette couche perd progressivement de sa puissance en allant vers le sud-est du profil, et contrairement en se dirigeant vers sa partie nord-ouest. Elle recouvre fidèlement la couche 6 . Elle est continue jusque dans la zone B, profil 2 de ce locus. Le sédiment a une texture sablo-argilo-limoneuse, emballant quelques concrétions libres de petites et moyennes dimensions. Il est relativement peu compact, non structuré, et de couleur jaune brunâtre.

Couche 2 : elle prolonge sous le dépôt de la couche $1 \mathrm{du}$ profil 2 sur une épaisseur de $50 \mathrm{~cm}$ environ. Elle est composée de cailloutis et de blocs calcaires de taille variable, emballés dans une matrice sablo-limono-argileuse de couleur jaune. Le dépôt est assez consolide et non structure. Le sédiment fin est collant, plastique à l'état humide.

Couche 1 : elle est développée dans cette zone sur une épaisseur d'un mètre environ. Il s'agit d'un ensemble de niveaux sablo-limono-argileux à cailloutis et blocs calcaires de grandes tailles. Le sédiment fin est de couleur jaune brunâtre et assez consolidé.

\section{Secteur I, profil 2, zone B}

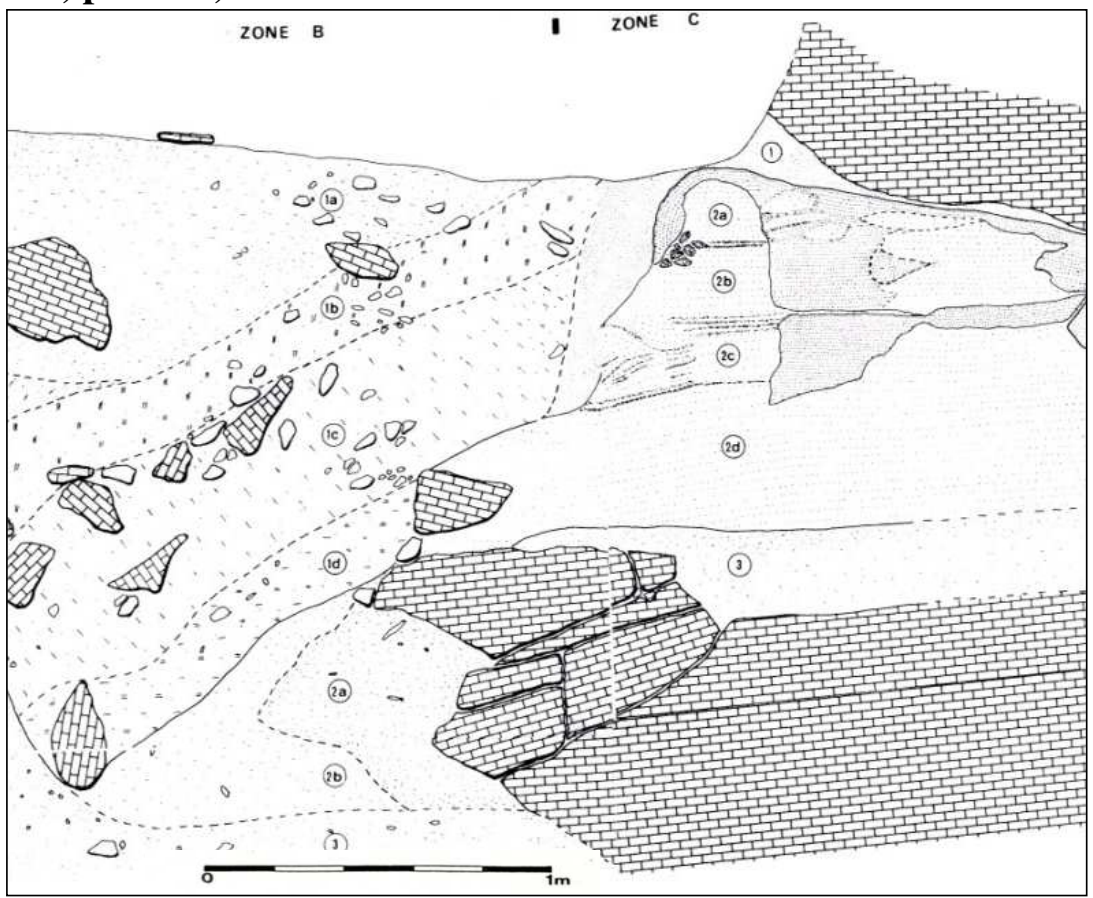

Fig. 5 : Coupe stratigraphique des deux zones B et C du secteur I. 
On distingue de bas en haut les unités stratigraphiques suivantes :

Couche 3 : cette couche est visible sur une épaisseur de $30 \mathrm{~cm}$ environ. Sa base n'a pas été atteinte. Elle prolonge sous la couche 3 décrite au niveau du profil 1. Le sédiment qui la constitue présente une texture sablo-limono-argileuse. Il emballe quelques concrétions libres de petites et moyennes dimensions. Il est peu compact et de couleur jaune brunâtre.

Couche 2: subdivisée en deux niveaux: niveaux $2 \mathrm{a}$ et $2 \mathrm{~b}$. D'épaisseur maximale $1 \mathrm{~m}$, cette couche repose sur la couche 3 et se présente sous forme d'une lentille. Il s'agit d'un dépôt de sables siliceux à micas blanc, non structuré, peu compact qui semble avoir fait l'objet de remaniements post dépôt.

Niveau 1d: d'épaisseur maximale $35 \mathrm{~cm}$, c'est un sédiment de texture limono-sablo-argileuse, à cailloutis et blocs calcaires de dimensions variables. Le sédiment fin est de couleur jaune, assez consolidé à l'état sec.

Niveau 1c: d'épaisseur variant de 60 à $90 \mathrm{~cm}$, ce niveau surmonte directement le niveau précédent. Le sédiment fin présente à peu prés les mêmes caractéristiques que celles du niveau 1d. Cependant, il est relativement plus chargé en cailloux calcaires de tailles moyennes.

Niveau $1 \mathrm{~b}$ : son épaisseur varie de 30 à $40 \mathrm{~cm}$ au maximum. Le dépôt de ce niveau est très comparable à ceux qui caractérisent les niveaux sousjacents (1c et 1d). Il se distingue par une couleur légèrement plus foncée, une matrice limono- sablo-argileuse et une moindre teneur en blocs carbonatés de grandes dimensions.

Niveau 1a : il fait le prolongement du dépôt supérieur de la couche 1 du profil 1. Il est composé par un sédiment de texture sablo-limonoargileuse. Cependant, il diffère du dépôt sous-jacent par sa couleur jaune brunâtre. Ce dépôt est assez consolidé et non structuré.

\section{Secteur I, profil 2, zone C}

Dans cette zone, la nature des dépôts rappelle celle des dépôts du secteur II du premier étage. On distingue de bas en haut les unités stratigraphiques suivantes :

Couche 3 : elle est visible sur une épaisseur de $40 \mathrm{~cm}$ au maximum. Cette couche s'appuie directement sur les blocs carbonatés qui délimitent ce que l'on appelle le premier étage. Le sédiment qui forme cette couche est de texture limono- argilo-sableuse, peu compact et de couleur jaune brunâtre. Sa limite inférieure est nette et régulière.

Couche 2: de puissance maximale $1,5 \mathrm{~m}$, cette couche est une formation de sables siliceux à mica blanc, renfermant quelques granules quartzeux de petites dimensions. Au sein de cette formation sableuse, existent des horizons concrétionnés qui la subdivisent en niveaux 
d'épaisseurs inégales, ce qui lui donne un aspect lité, notamment dans sa partie supérieure.

Couche 1 : elle a été érodée dans son quasi totalité. Elle surmonte directement le banc gréseux formé par le concrétionnement d'une partie de la formation sableuse sous-jacente. Il s'agit d'un dépôt limoneux assez compact et de couleur jaune à brun très pâle, de structure originelle finement litée. Ce sédiment est fortement remanié dans sa plus grande partie.

\section{Etude sédimentologique}

\section{Profil 1 (zone A)}

\section{Diagramme stratigraphique global}

Le diagramme granulométrique global met en évidence deux ensembles sédimentaires bien distincts :

Ensemble inférieur: Il comprend les couches 10 à 4. La fraction grossière est absente de tous les niveaux de cet ensemble. Les éléments de diamètre supérieur à $2 \mathrm{~mm}$ sont représentés par des granules quartzeux dans la couche 6 et les niveaux 10a et 10b. Cependant, la fraction fine constitue $5 \%$ du sédiment total dans la couche 6. Dans les niveaux 10a et 10b, elle entre respectivement pour 6 à $19 \%$ dans la composition de l'ensemble du matériel analysé.

Ensemble supérieur: Il regroupe les couches 3 à 1 . Il se caractérise par l'apparition des éléments de diamètre supérieur à $10 \mathrm{~mm}$. En effet, la fraction grossière entre pour $35 \%$ dans la formation du matériel provenant de la couche 2. Dans les niveaux 1c, 1b et 1a, les éléments grossiers de dimensions supérieures à $10 \mathrm{~mm}$ entrent respectivement pour 47,15 et $20 \%$ de la composition globale des sédiments. D'une manière générale, on remarque que l'enrichissement des sédiments en éléments grossiers s'accompagne d'une augmentation de la taille de ces éléments, comme en témoigne les variations du taux de la fraction grossière. Cette dernière forme 45 à $90 \%$ des éléments de diamètre supérieur à $2 \mathrm{~mm}$. Cette grossièreté des éboulis est particulièrement nette dans la couche 2 et au sommet de la couche 1.

\section{Etude de la fraction grossière Granulométrie des cailloux}

Les cailloux non concrétionnes sont présents dans la partie supérieure de la couche 1 et dans la couche 2. Dans la couche 3, ils ne sont que faiblement représentés et ne dépassent pas $2 \mathrm{~cm}$ de diamètre. En revanche, les cailloux constituent $45 \%$ de la fraction grossière de la couche 2 . Leur diamètre varie de 1 à $4 \mathrm{~cm}$. Dans la partie supérieure de la couche 1 , les cailloux ne dépassent pas $3 \mathrm{~cm}$ de diamètre. 


\section{Analyses morphoscopiques}

\section{Actions de gel}

Les manifestations secondaires de gel sont très peu marquées dans ces dépôts, à l'exception de la couche 2 ou les cailloux fissurés constituent prés de $34 \%$ de la phase caillouteuse. Les plaquettes de gel à arêtes assez émoussées sont rencontrées également dans cette couche, bien que leur taux reste faible et ne dépasse pas $10 \%$ de l'ensemble des cailloux.

\section{Actions de l'humidité}

Cailloux concrétionnés : ils sont présents dans tous les niveaux de la couche 1 . Ils forment $53 \%$ des éléments de diamètre supérieur à $10 \mathrm{~mm}$ dans le niveau 1c. Leur proportion diminue progressivement en allant vers le sommet de cette couche pour ne composer que $13 \%$ de la fraction grossière. Dans la couche 3, les cailloux concrétionnés entrent pour $61 \%$ dans la composition de la fraction grossière. Ils font défaut dans la couche 2.

Concrétions illuviales : cette catégorie de concrétions est développée dans tous les niveaux à éléments grossiers carbonatés et entre dans la composition de toutes les fractions grossières et moyennes. En effet, ces concrétions forment prés de $19 \%$ des éléments dont le diamètre est supérieur à $2 \mathrm{~mm}$ au niveau de la couche 2 . Elles constituent $11 \%$ du matériel grossier dans la couche 1 . Elles entrent pour plus de $73 \%$ dans la composition de la fraction grossière du niveau $1 b$ et $74 \%$ des granules de ce même niveau. Elles sont moins développées dans la couche 3 puisque leur taux ne dépasse pas $2 \%$ par rapport à l'ensemble du sédiment. La morphologie de ces concrétions est la même dans tous les échantillons analysés. Il s'agit d'agrégats de sédiment fin cimentés par un ciment calcaire. Ces agrégats sont très durs et montrent souvent des microcavités de dissolution.

\section{Etude de la fraction fine}

\section{Diagramme granulométrique global}

Ce diagramme confirme l'existence des deux ensembles mis en évidence par le diagramme stratigraphique global. Dans le détail, on distingue :

- un ensemble inférieur : qui correspond aux couches 10 à 4 dans lesquelles, les trois composantes (sables, limons et argiles) montrent des variations de grande amplitude. On note tout d'abord un enrichissement des sédiments en fraction sableuse dans les niveaux 10a et $10 \mathrm{~b}$ ou les sables forment respectivement 76 et $90 \%$ du matériel inférieur à $2 \mathrm{~mm}$. En revanche, ils ne constituent que prés de $29 \%$ du dépôt provenant de la couche 10. Leur proportion augments ensuite dans la couche 9 pour atteindre $74 \%$ du sédiment total. Dans la couche 8 , le taux des sables ne dépasse pas $22 \%$ du matériel fin. Il présente sa valeur maximale dans la couche 7 avec 
$95 \%$. Le pourcentage des sables diminue de nouveau jusqu'à dans la couche 4 pour ne former que $39 \%$ du sédiment fin.

Les limons sont moins importants que les sables sauf dans la couche 10 ou ils constituent $42 \%$ du dépôt. Dans les autres couches, les limons n'excédent jamais $25 \%$. Ils entrent respectivement pour 25 et $24 \%$ dans la composition des sédiments provenant des couches 5 et 4 . Leur taux le plus faible est enregistré dans la couche 7 et $10 \mathrm{~b}$ avec seulement $4 \%$.

Le pourcentage des argiles varie de façon inverse à celui des sables. Leur proportion la plus grande s'enregistre dans la couche 8 où elles entrent pour $60 \%$ dans la composition de cette couche. Les argiles sont également bien représentées dans les couches 10,5 et 4 et constituent respectivement 28, 22 et $37 \%$ de leur matériel inférieur à $2 \mathrm{~mm}$. Dans les autres couches, la fraction argileuse ne dépasse pas $15 \%$.

- un ensemble supérieur: qui regroupe les couches 3 à 1 . Dans cet ensemble, les coupures stratigraphiques mise en évidence par le diagramme granulométrique sont moins importantes que dans les dépôts de l'ensemble inférieur. Les sables entrent pour 20 à $39 \%$ dans la constitution du sédiment fin. Leur pourcentage augmente légèrement de la couche 3 (34 \%) jusqu'a la base de la couche 1 (niveau 1c) pour atteindre sa valeur maximale au sein de cet ensemble. Il diminue ensuite sensiblement au niveau $1 \mathrm{~b}$ et au niveau $1 \mathrm{a}$ où les sables constituent respectivement 20 et $22 \%$ de la fraction fine.

Les limons sont plus abondants que dans l'ensemble inférieur, et forment 27 à $45 \%$ de la fraction fine. Leur taux le plus élève est enregistré dans les niveaux $1 \mathrm{a}$ et $1 \mathrm{~b}$.

On constate une légère illuviation d'argiles dans la couche 3 où elles sont les mieux représentées avec prés de $40 \%$. Dans les autres niveaux, elles entrent pour 26 à $35 \%$ dans la composition du sédiment fin.

\section{Calcimétrie}

La courbe des variations de la teneur en carbonates fait apparaitre deux parties distinctes dans ce profil :

Une partie inférieure regroupant les couches 10 à 5 où les carbonates entrent pour une faible part dans la composition de sédiments fins. Leur taux varie alors de 5 à $13 \%$ à l'exception de la couche 8 ou on enregistre tout de même $19 \%$ de carbonates dans la fraction fine.

En revanche, dans la partie supérieure composée des couches 4 à 1 , les proportions de carbonates sont nettement plus importantes dans tous les échantillons examinés. On remarque notamment un enrichissement de sédiments fins en carbonates dans la couche 3 où on enregistre leur pourcentage le plus élevé de cette partie du remplissage avec $42 \%$. A part quelques fluctuations mineures, leur valeur diminue de façon à peu prés progressive en allant vers le sommet du profil pour ne former que 22 à $24 \%$ 
du matériel fin dans les niveaux $1 \mathrm{~b}$ et 1a. Cette évolution peut être expliquée par une accumulation illuviale des carbonates, par lessivage à partir des niveaux supérieurs. La comparaison des diagrammes granulométriques globaux des sédiments fins avant et après décarbonatation montre que les carbonates sont stockés plus particulièrement dans les fractions sabloargileuses, et cela dans les couches supérieures riches en carbonates, tandis que dans les couches inférieures pauvres en carbonates, ces derniers sont emmagasinés notamment dans la phase argileuse.

\section{Morphoscopie des grains de quartz.}

La répartition des grains de quartz en classes morphoscopiques permet de suivre les variations des différentes catégories rencontrées dans cette série d'échantillons. En effet, les grains sub-anguleux luisants présentent des fluctuations assez importantes. Dans la couche 10, ils constituent $30 \%$ du cortège quartzeux. Ils se développent dans la couche 9 (53\%). Dans la couche 8, les grains sub-anguleux luisants entrent pour $40 \%$ dans la composition des grains de quartz. Leur proportion augmente dans les couches 7 et 6 où ils forment environ $55 \%$ du cortège quartzeux. Ils décroissent de nouveau jusque dans la couche 4 où leur pourcentage ne dépasse pas $30 \%$, pour augmenter ensuite dans la couche 3 avec $46 \%$. Les échantillons des couches 2 et 1 renferment une proportion assez constante de ce type de grains dont le taux varie de 26 à $30 \%$. Les grains sub-anguleux à peine mats sont bien représentés dans la couche 10 (42\%). Dans les couches 9 à 5, leur pourcentage vari très peu et oscille entre 24 et $32 \%$. Les échantillons provenant des couches 4 à 1 renferment 36 à $40 \%$ de cette catégorie de grains de quartz. Les grains non usés montrent moins de variations dans cette série. Ils constituent prés de $12 \%$ dans la couche 10 . Leur taux augmente faiblement jusque dans la couche 8 (20\%) pour diminuer à nouveau et de façon progressive jusqu'au niveau de la couche 3 où on enregistre leur valeur la plus faible de ce profil $(8 \%)$. Dans les couches 2 et 1 , ils entrent pour 22 à $28 \%$ dans la composition de leur cortège quartzeux. Les grains arrondis mats sont peu nombreux sauf dans la couche 4 où ils atteignent leur valeur maximale $(20 \%)$. Par ailleurs, ils ne dépassent jamais $12 \%$ des grains analyses.

\section{Profil 2 : Zone B}

\section{Diagramme stratigraphique global}

Dans la couche 3 : le sédiment apparait moyennement chargé en éléments grossiers. La fraction grossière constitue $37 \%$ de l'ensemble du matériel analysé. Elle n'est composée pratiquement que de concrétions illuviales. Les fractions moyennes sont faiblement représentées et forment moins de $5 \%$ du sédiment total. 
Au niveau de la couche 2 : le dépôt est constitué essentiellement de sédiment inférieur à $2 \mathrm{~mm}$. Les granules ne sont que peu développés et entrent pour environ $7 \%$ au maximum dans la composition du matériel global.

Dans la couche 1 : tous les niveaux sont chargés d'éléments grossiers de diamètre compris entre 2 et $100 \mathrm{~mm}$ qui entrent pour 45 à $61 \%$ dans la constitution des sédiments. Cependant, la fraction grossière reste largement dominante par rapport aux fractions fines; elle représente 80 à $90 \%$ des éléments de diamètre supérieur à $2 \mathrm{~mm}$. Les fractions fines varient de façon parallèle l'une par rapport a l'autre. C'est dans le niveau 1c qu'elles sont les moins représentées et composent respectivement 5 et $4 \%$ de l'ensemble du sédiment.

\section{Etude de la fraction grossière}

Les fractions grossières sont composées uniquement de concrétions, sauf dans le niveau 1a où on rencontre tout de même des cailloux carbonatés non concrétionnés. Ces derniers constituent prés de $21 \%$ des éléments grossiers de ce niveau. Ils sont généralement émoussés ou fortement altérés. Dans la couche 3, les concrétions sont formées exclusivement de concrétions illuviales. De même dans les niveaux $1 \mathrm{~d}$ et $1 \mathrm{c}$, ce type de concrétions compose la quasi totalité des éléments grossiers. Dans les niveaux 1a et $1 \mathrm{~b}$, les concrétions sont de deux aspects : des cailloux concrétionnés qui forment respectivement 24 et $14 \%$ du sédiment total, mais également des concrétions illuviales qui entrent pour 30 et $13 \%$ dans la composition de l'ensemble des sédiments.

\section{Etude de la fraction fine}

\section{Diagramme granulométrique global}

Ce diagramme montre des fluctuations assez marquées. Dans la couche 3, la fraction sableuse forme $30 \%$ du sédiment fin. Elle est bien représentée dans les deux niveaux de la couche 2 puisqu'elle constitue 71 à $78 \%$ de leur matériel fin. En revanche, les proportions des sables ne dépassent jamais $50 \%$ de la fraction fine provenant des niveaux $1 \mathrm{~d}$ à $1 \mathrm{a}$. C'est dans les niveaux 1d et 1c que les sables sont les moins développés. Leur pourcentage varie de 30 é $32 \%$ par rapport au sédiment fin. La fraction limoneuse varie dans le sens inverse de celui de la fraction sableuse. Elle est maximale dans la couche 3 avec $23 \%$ du sédiment fin et minimale dans la couche 2 où elle entre pour 6 à $9 \%$ dans la formation de son matériel fin. Les limons sont assez abondants au niveau 1d (30\%). Leur taux diminue ensuite jusqu'au niveau 1 a pour ne former que $19 \%$ du sédiment inférieur à $2 \mathrm{~mm}$. 
Les argiles évoluent de façon parallèle par rapport aux limons. Elles sont assez bien représentées dans la couche 3 (36\%). Elles sont peu abondantes dans les deux niveaux de la couche 2 où elles constituent 16 à 19 $\%$ du matériel fin. Dans les niveaux de la couche 1, leur pourcentage oscille entre 30 et $42 \%$ avec une illuviation au niveau $1 \mathrm{c}$.

\section{Calcimétrie}

Le taux des carbonates le plus élevé de cette série est enregistré au sommet de la couche 1 (niveau 1a) ou il atteint prés de $48 \%$. Le pourcentage des carbonates diminue ensuite jusqu'à la base de la couche 1 (niveau 1d) pour ne constituer que $30 \%$. Dans les deux niveaux de la couche 2, les carbonates ne sont que faiblement représentés puisqu'ils composent seulement 5 et $10 \%$ du sédiment fin. Dans la couche 3 , on note à nouveau un léger enrichissement en carbonates avec $22 \%$ du matériel analysé. La comparaison des deux diagrammes globaux de sédiments fins avant et après décarbonatation montre que ces carbonates sont présents dans les particules argileuses, mais surtout dans les fractions sableuses au sein des dépôts supérieurs de cette partie du remplissage (couche 1).

\section{Profil 2 : Zone C}

\section{Diagramme stratigraphique global}

Ce diagramme montre que les couches 1 et 3 sont dépourvues d'éléments grossiers de diamètre supérieur à $2 \mathrm{~mm}$. Les niveaux sableux de la couche 2 renferment quelques granules dont les dimensions ne dépassent pas $5 \mathrm{~mm}$ et dont les proportions varient de 5 à $13 \%$ au maximum.

\section{Etude de la fraction fine}

Couche 3 : le sédiment de cette couche est composé de $50 \%$ de limons, $23 \%$ de sables et $27 \%$ d'argiles. Sa représentation graphique montre une grande ressemblance avec celle qui caractérise la couche 3 de la zone B de ce même profil. Enfin, le sédiment de cette couche ne renferme que $12 \%$ de carbonates.

Couche $2:$ les caractéristiques sédimentologiques des sédiments fins provenant de cette couche sont remarquablement comparables à celles des matériaux fins de la couche 3 du secteur II du premier étage. Les sables grossiers sont largement dominants dans tous les niveaux par rapport aux autres fractions fines et constituent 60 à $77 \%$ des sédiments tamisés à $2 \mathrm{~mm}$. Après traitement à l'acide chlorhydrique, les sédiments se repartissent en des courbes cumulatives très voisines de celles des sédiments bruts, ce qui est normal, puisque le taux des carbonates n'excède jamais $8 \%$. Ces courbes granulométriques témoignent alors des mêmes caractéristiques que celles des matériaux bruts. 
Couche 1 : Le sédiment de cette couche est composé essentiellement de limons (88\%). Les sables et les argiles ne forment que 10 et $2 \%$ de ce dépôt. La composante carbonatée entre pour $18 \%$ dans la formation de ce dépôt.

\section{Morpho scopie des grains de quartz}

Dans cette série d'échantillons, ce sont les grains subi-anguleux luisants qui dominent dans les niveaux sableux de la couche 2. Leur pourcentage varie de 58 à $68 \%$. Cette catégorie de grains constitue également prés de $41 \%$ du cortège quartzeux provenant de la couche 3 .

- Les grains sub-anguleux à peine mats forment 18 à $22 \%$ des quartzs de sable de la couche 2 et environ $28 \%$ de ceux de la couche 3 .

- Les grains émoussés luisants sont peu nombreux et entrent pour 3 à $10 \%$ dans la composition des quartzs examinés.

- Les grains non usés sont présents dans tous les échantillons et leur valeur oscille entre 10 et $18 \%$.

\section{Variations latérales des faciès}

Les observations effectuées sur le terrain ainsi que l'étude de trois séries d'échantillons apportent quelques précisions sur les variations latérales dans cette partie du remplissage. En effet, la comparaison des diagrammes granulométriques globaux montre que les éléments grossiers, dont la taille ne dépasse pas $100 \mathrm{~mm}$ de diamètre, sont plus nombreux dans la zone $\mathrm{B}$ que dans la zone $\mathrm{A}$, et cela dans les dépôts carbonatés à cailloutis (couches 1 et 2 de la zone A, et couche 1 de la zone B). Dans la couche 3 des zones A et B, les éléments grossiers de diamètre supérieur à $2 \mathrm{~mm}$ forment $42 \%$ du sédiment total dans la zone $\mathrm{B}$, alors qu'ils n'excédent pas $18 \%$ au niveau de la zone A.

De point de vue morphologie des éléments grossiers, les concrétions illuviales sont mieux représentées dans la zone B que dans la zone A. En effet, cette catégorie de concrétions constitue la totalité des éléments de diamètre supérieur à $2 \mathrm{~mm}$ de la couche 3 et des niveaux $1 \mathrm{c}$ et $1 \mathrm{~d}$ de la zone B. Dans les niveaux supérieurs de la couche 1, ils forment plus de $40 \%$ des fractions grossières. En revanche, dans la zone A, ces concrétions perdent de leur importance au profit des cailloux carbonatés. Dans la couche 1, on remarque également un enrichissement des sédiments fins en fraction limoneuse au niveau de la zone $\mathrm{B}$ par rapport à la zone $\mathrm{A}$. Au contraire, dans la couche 3 , les limons perdent de leur importance en allant de la zone A à la zone $\mathrm{C}$. 


\section{Interprétations dynamique et climatique des dépôts}

Au niveau du profil 1, les couches 10 à 3 (ensemble inferieur) correspondent vraisemblablement à un climat tempéré et humide, cela en raison de l'absence des éléments grossiers carbonatés d'origine cryoclastique. La structure litée de ces dépôts et la prédominance des grains quartzeux luisants dans les sédiments montrent que ces matériaux ont été transportés et mis en place par l'eau. La présence de sels de manganèse dans ces sédiments et l'absence de grains de quartz franchement mats apportent une confirmation à cette interprétation. D'autre part, l'intensité et le régime des ruissellements avaient certainement une influence déterminante sur les caractéristiques sédimentologiques de ces sédiments. Ainsi, le dépôt de la couche 10 semble être transporté par ruissellement de faible intensité et mis en place dans des eaux calmes, comme en témoignent sa structure finement litée et sa texture fine. Le caractère plurimodal de sa courbe cumulative traduit un apport par pulsations et non pas continu. L'importance prise par la fraction limoneuse dans cette couche peut être expliquée par une faible éolisation des dépôts dont ils ont été hérités. Pour les dépôts qui représentent les niveaux 10a et $10 \mathrm{~b}$, la texture grossière et le bon classement des sédiments témoignent d'une mise en place par ruissellement plutôt soutenu. Ce qui explique le ravinement du dépôt de la couche 10.

Dans le dépôt de cet ensemble, on remarque que les sédiments sont d'autant plus classés que leur grossièreté moyenne est élevée. Ce qui indique que cette caractéristique est liée vraisemblablement au régime et à l'intensité des ruissellements qui les ont transportés. Dans le cas de la couche 7, les ruissellements ont entrainé une grande partie des particules les plus fines par lessivage, ce qui a pour effet d'augmenter la grossièreté de son sédiment. Les sédiments de la couche 3 ont été déposés par ruissellements diffus et sous un climat moins humide que celui qui a présidé à la mise en place du dépôt sous-jacent (couche 6 et 7). Le dépôt de cette couche a été par ailleurs en partie remanié postérieurement à son édification. Une composante carbonatée essentiellement argileuse vient s'ajouter à ces sédiments par illuviation à partir des dépôts supérieurs, notamment dans les couches 3 et 4 . Au niveau du profil 2, la mise en place des dépôts sableux qui composent la couche 2 des zones $\mathrm{B}$ et $\mathrm{C}$ demande également des conditions climatiques tempérées et relativement plus humides que celles qui ont présidées à l'édification de la couche 3 de la zone $\mathrm{C}$. Ces épisodes humides ont du être interrompues par des périodes assez sèches qui ont permis l'encroûtement d'une partie de ces sables qui se transforment en un véritable banc gréseux dans la partie nord-ouest de ce secteur (zone C). Les deux niveaux de la couche 2 de la zone B ont fait l'objet d'un remaniement post sédimentaire. $\mathrm{Ce}$ processus de remaniement a joué un rôle prépondérant dans le démantèlement des niveaux concrétionnés, et par conséquent dans la 
disparition de leur structure litée originelle. Cette structure s'observe toujours dans la couche 2 de la zone $\mathrm{C}$. Au dessus de ces sables lités, le dépôt limoneux qui forme la couche 1 dans la zone $\mathrm{C}$ est transporté par ruissellement de faible compétence, et mis en place dans des eaux calmes comme en témoigne sa structure originelle laminaire. Après l'édification des couches à sédiments essentiellement allochtones, les dépôts carbonatés des couches 1 et 2 de la zone A et ceux de la couche 1 de la zone B sont mis en place par éboulements. Par conséquent, ces derniers dépôts ne sont pas en place archéologiquement.

\section{Secteur II}

\section{Stratigraphie (fig. 6 et 7)}

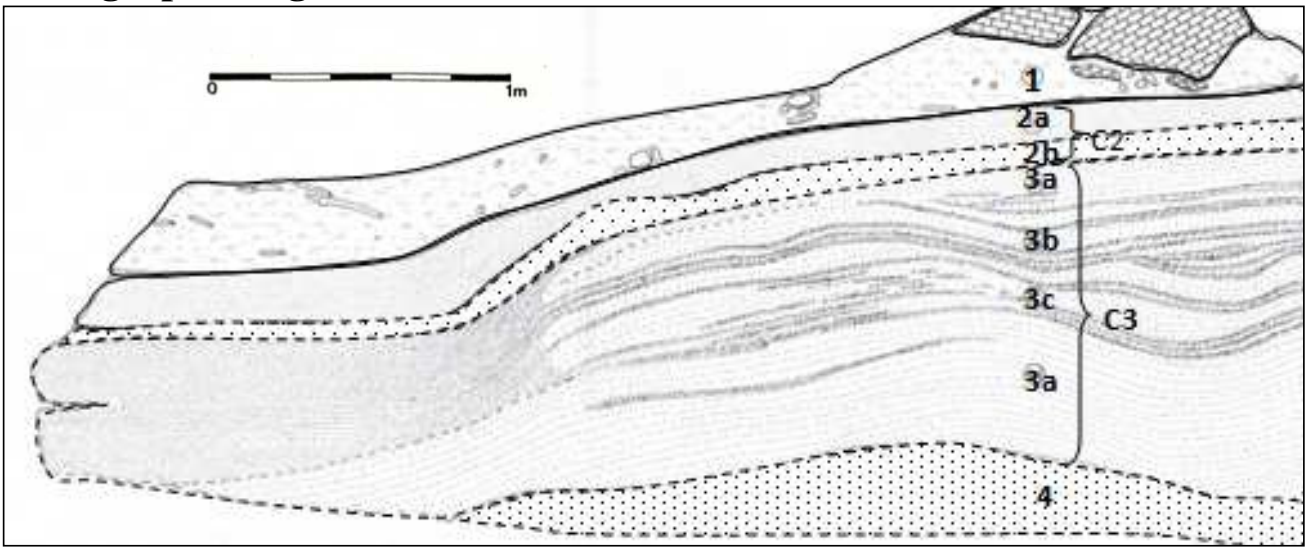

Fig. 6 : coupe stratigraphique, premier étage, secteur II : (série 1)

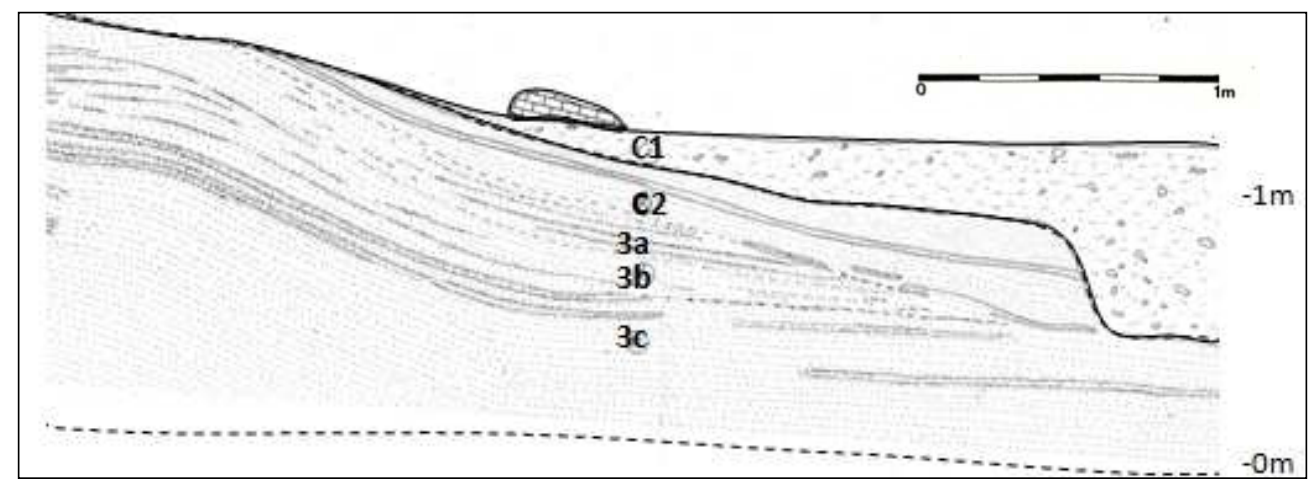

Fig. 7 : coupe stratigraphique, premier étage, secteur II (série 2)

Dans ce secteur, on reconnait de bas en haut les unités stratigraphiques suivantes:

Couche 4 : elle est visible sur une puissance d'environ $50 \mathrm{~cm}$. Sa base n'a pas été atteinte. Il s'agit d'un dépôt assez fin, de texture sablo-limonoargileux, renfermant des ossements. Le sédiment est peu compact, remanié 
dans sa grande partie et de couleur jaune brunâtre. La limite inférieure de cette couche est nette et assez régulière.

Couche 3: d'épaisseur maximale 1,5 m, c'est une importante formation sableuse, étendue sur plus de 10 mètres. Elle se développe sous forme de niveaux de sables siliceux à mica blanc emballant quelques rares granules de quartz et de quartzites de petites dimensions. L'aspect nettement lité de cette formation est dû au développement d'encroutements rubanés de quelques millimètres a quelques centimètres d'épaisseur, plus ou moins continus. Ces horizons concrétionnés séparent des niveaux à sédiments de même texture, peu compacts, friables et épais de quelques centimètres. Ce phénomène de concrétionnement affecte cette formation sableuse sur toute sa puissance vers sa partie est et transforme ces matériaux en un véritable banc gréseux. Il faut signaler également l'édification d'une dalle gréseuse très compacte constituée par des horizons d'encro0tement, extrêmement durs, épaisse de $8 \mathrm{~cm}$. Cette dalle s'étend sur toute la longueur de la formation sableuse à sa partie supérieure. Dans la zone médiane de la coupe, le pendage de ces lits sableux varie de la base au sommet de ce dépôt sableux. Il est de l'ordre de 40 à $45^{\circ}$ Nord à la base, puis perd progressivement de son inclinaison en allant vers les niveaux les plus récents pour s'annuler à son sommet.

Couche 2 : elle se subdivise en deux niveaux :

Niveau 2a : d'épaisseur moyenne $15 \mathrm{~cm}$, il devient progressivement plus important en allant vers la partie ouest de la coupe où il est tronqué par le dépôt de la couche 1. Ce niveau est composé essentiellement de limons. Le sédiment est compact, de structure laminaire fine et montre des traces de bioturbation (terriers). A l'état sec, il est de couleur gris clair. Niveau $2 \mathrm{~b}$ : d'épaisseur moyenne $5 \mathrm{~cm}$, il présente à peu prés les mêmes caractéristiques stratigraphiques que le niveau 2 a (texture, structure, consistance). Cependant, il se différencie par sa couleur jaune. Sa limite inferieure est nette et régulière.

Couche 1 : elle s'observe sur une épaisseur maximale de $45 \mathrm{~cm}$. Elle est tronquée au niveau de la partie médiane de la coupe. Cette couche est formée de sédiment fin, de texture sablo-limono-argileuse, emballant quelques cailloutis et blocs carbonatés de tailles variables. Le matériel fin est peu compact et de couleur brun très pâle.

\section{Etude sédimentologique de la série 1}

\section{Diagramme stratigraphique global} différents :

Ce diagramme permet de subdiviser ces dépôts en trois ensembles

Ensemble inferieur: il correspond aux couches 4 et 3. Dans cet ensemble, la fraction grossière est absente de tous les niveaux. Les éléments 
grossiers sont constitues uniquement de granules. C'est dans le niveau $3 b$ que les fractions fines sont les plus représentées, bien que leur proportion n'excède pas $11 \%$ de l'ensemble du matériel analysé.

Ensemble moyen : il regroupe les deux niveaux de la couche 2 et se compose exclusivement de sédiments fins.

Ensemble supérieur: il est formé par la couche 1. Il renferme quelques éléments grossiers. La fraction grossière entre pour $15 \%$ dans la constitution $\mathrm{du}$ sédiment total. Les fractions fines sont faiblement représentées et ne dépassent pas $1 \%$ dans ce dépôt.

\section{Etude de la fraction fine}

\section{Diagramme granulométrique global}

La fraction sableuse entre pour une part importante dans la constitution du matériel de la couche 4 puisqu'elle forme prés de $51 \%$. Dans les quatre échantillons de la couche 3 (niveaux $3 d, 3 c, 3 b$ et $3 a$ ), les sables sont largement majoritaires et composent 75 à $88 \%$ du matériel tamisé à 2 $\mathrm{mm}$. Au niveau de la couche 2, ils sont faiblement représentés puisqu'ils constituent moins de $10 \%$ du sédiment dans le niveau $2 \mathrm{~b}$ et seulement $20 \%$ au niveau 2a. Enfin, leur proportion n'excède guère $8 \%$ dans la fraction fine de la couche 1 .

Les limons constituent environ $13 \%$ dans la couche 4 . Ils sont pratiquement absents à la base de la couche 3 (niveau 3d). Leur taux augmente légèrement en allant vers le sommet, mais ne dépasse pas $6 \% \mathrm{du}$ sédiment inferieur à $2 \mathrm{~mm}$. Dans les niveaux $2 \mathrm{~b}$ et $2 \mathrm{a}$, les limons entrent respectivement pour 88 et $78 \%$ dans la formation des sédiments fins. Ils sont moins abondants dans la couche 1 ou leur pourcentage est de l'ordre de 54 $\%$.

Les argiles représentent $35 \%$ du matériel fin provenant de la couche 4. Elles ne constituent cependant que 12 à $19 \%$ des fractions fines des niveaux $3 \mathrm{~d}$ à $3 \mathrm{a}$. La fraction argileuse de la couche 2 représente moins de 2 $\%$ du dépôt dans les niveaux a et $b$. C'est dans la couche 1 que les argiles atteignent leur valeur maximale $38 \%$ du matériel fin.

\section{Calcimétrie}

Le taux des carbonates est faible dans cette partie du remplissage. Les carbonates entrent pour moins de $10 \%$ dans la composition du sédiment de la couche 4 . Leur proportion n'excède jamais $5 \%$ dans les niveaux de la couche 3 . Dans les niveaux $2 \mathrm{~b}$ et $2 \mathrm{a}$, ils constituent respectivement 14 et 20 $\%$ de leur matériel. Au niveau de la couche 1, on enregistre prés de $12 \%$ de carbonates dans la fraction fine. 


\section{Morphoscopie des grains de quartz}

L'étude morphoscopique des grains de quartz montre la dominance des grains sub-anguleux luisants dans tous les niveaux sableux de la couche 3 où ils entrent pour 66 à $71 \%$ de la composition du cortège quartzeux. Dans la couche 4 , cette catégorie de grains de quartz constitue prés de $50 \%$ des grains examinés. Les grains sub-anguleux à peine mats sont assez abondants dans la couche $4(25 \%)$. Leur proportion est pratiquement constante dans la couche 3 où ils constituent 21 à $23 \%$ des quartzs de diamètre compris entre 0,5 et $0,8 \mathrm{~mm}$. Les grains sub-arrondis à peine mats sont présents dans les couches 4 et 3 avec un taux de $10 \%$.

\section{Etude sédimentologique de la série 2} Diagramme stratigraphique global

Ce diagramme fait apparaitre les mêmes caractéristiques granulométriques que celles révélées par celui qui caractérise la série 1. On constate tout de même une légère diminution de la proportion des éléments grossiers dans la couche 1 dans cette zone de la coupe, puisqu'ils ne forment que 6\% du matériel total analysé. Dans le cas de la couche 3, les variations texturales sont moins sensibles dans les trois niveaux supérieurs.

\section{Etude de la fraction fine}

\section{Diagramme granulométrique global}

Ce diagramme met en évidence d'importantes variations latérales au niveau de la couche 1 . On remarque que le matériel tamisé à $2 \mathrm{~mm}$ devient encore plus fin en allant de l'Est à l'Ouest de ce profil. Cette finesse de sédiment se traduit par l'augmentation du taux des argiles, et cela au détriment des limons qui restent, cependant, prédominants par rapport aux autres fractions fines. En effet, la fraction argileuse constitue $55 \%$ de la fraction fine au lieu de $38 \%$ au niveau de la série 1 . En ce qui concerne la couche 3 , ce diagramme montre une grande ressemblance texturale entre les échantillons quelle que soit leur provenance au sein de cette coupe. Les variations latérales y sont pratiquement nulles. En conclusion, les variations latérales de la texture du sédiment dans la couche 1 peuvent avoir deux origines :

- une origine primaire liée à un granoclassement latéral des matériaux lors de leur mise en place ; argileuses.

- une origine secondaire liée à une illuviation latérale des particules

\section{Calcimétrie}

Les carbonates sont également peu développés dans tous les échantillons provenant de la couche 3 ou leur taux n'excède pas $5 \%$. Dans 
les couches 1 et 2 , ils constituent respectivement 14 et $16 \%$ du matériel inférieur à $2 \mathrm{~mm}$. On signale également que ces carbonates sont stockés particulièrement sous forme des particules argileuses.

\section{Etude du banc gréseux Etude macroscopique}

Ce banc résulte du concrétionnement de la partie Ouest des niveaux de sables qui constituent la couche 2 (série C, secteur I), mais également de la zone Est de la formation sableuse qui forme la couche $3 \mathrm{du}$ secteur II. Ce banc présente une structure litée et une texture sableuse plus ou moins grossière, avec des passages qui renferment quelques granules siliceux de petites dimensions. Des sédiments peu compacts et non concrétionnés se situent au sein de ce banc sous forme de petites lentilles, notamment dans le secteur I, zone C. Au niveau de ce banc gréseux, les niveaux à éléments grossiers sont débarrassés de leur matrice et le sédiment présente des vides intergranulaires bien visibles. En revanche, dans les niveaux à texture moyenne et fine, les grains de sables sont emballés dans une matrice où le ciment occupe une place prépondérante.

La dureté de ce banc est assez variable. A sa limite latérale avec les sédiments non consolidés, il est assez friable sous une faible pression des doigts. En contraire, il résiste aux coups de marteau dans les parties les plus dures.

De point de vue pétrographique, ce banc est composé essentiellement de grains quartzeux, imbriqués les uns dans les autres dans certains niveaux; ils sont dispersés dans une matrice de texture fine dans d'autres niveaux. Les granules rencontrés dans ce banc sont des quartzs et des quartzites, usés dans leur majorité. Des paillettes de muscovite qu'on peut observer sont déposées de façon aléatoire, sans aucune orientation par rapport aux litages de ce banc. Dans les niveaux à sédiment fin, les lamelles de mica blanc sont plus nombreuses et bien visibles à l'œil nu. Dans la zone $\mathrm{C}$ du secteur $\mathrm{I}$, le ciment argilo-carbonaté présente des traces d'oxydes de fer, ce qui donne un aspect pigmenté rouge brunâtre à certains niveaux affectés par ce type de liant.

\section{Etude microscopique des minéraux}

Deux lames ont été préparées à partir de deux échantillons provenant de ce banc ont fait l'objet d'une étude microscopique et qui montre les caractéristiques pétrographiques suivantes :

Lame $\mathrm{n}^{\circ} 1$ : l'étude au microscope de cette lame montre que la roche est constituée par l'entassement libre des grains sableux puisque ces derniers ne se rejoignent pas entre eux. Elle se caractérise alors par un squelette formé essentiellement de grains de quartz, associés à des minéraux feldspathiques, notamment des plagioclases. Ces derniers sont souvent très altérés. Parmi les 
minéraux légers qui entrent dans la constitution de cet échantillon, on trouve quelques éléments de muscovite mais également de la biotite. Les autres minéraux sont rares et dispersés de façon aléatoire. On reconnait toutefois des amphiboles, du chlorite, de la sillimanite, du disthène, des pyroxènes, du grenat et de la staurotide à l'état de traces. En ce qui concerne les quartzs, ils présentent dans leur majorité des contours sub-anguleux. Certains individus quartzeux montrent une extinction roulante, témoignant de contraintes tectoniques de la roche mère dont ils ont été hérites (Vatan A. 1956).

La présence de deux types de quartz : quartz à extinction droite et quartz à extinction roulante laisse envisager au moins une double origine des matériaux qui composent cette formation gréseuse. La matrice est formée d'un ciment calcitique à structure granulaire ou microgranulaire. Elle comble les espaces intergranulaires. Sa couleur est brune à brun foncé.

Lame $\mathrm{n}^{\circ} 2$ : la pétrographie montre une grande ressemblance avec celle de la lame 1. La seule différence réside dans la grossièreté de ses constituants : dans cet échantillon, les grains de quartz ont une taille plus importante, pouvant dépasser $2 \mathrm{~mm}$. Leur contour est également subanguleux, rarement sub- arrondi. Comme dans le cas de la lame précédente, les quartzs sont de deux types : des quartzs à extinction droite, mais également à extinction onduleuse. Les feldspaths sont moins représentés que les quartz et sont de dimension moyenne légèrement supérieure à celle des minéraux feldspathiques observés dans la première lame. La muscovite est également présente dans cette lame sous forme de paillettes plus ou moins allongées. Les autres minéraux sont très peu représentés. Ce sont essentiellement des amphiboles, de la biotite, de la chlorite, du disthène et de la staurotide. Il faut ajouter que l'on constate dans cette lame une diminution de l'espace intergranulaire par rapport à la lame $\mathrm{n}^{\circ} 1$. Cependant, les grains paraissent plus jointifs, laissant peu de place au ciment calcaire qui montre également par endroit une couleur rougeâtre, indiquant la présence de sels de fer imprégnés au sein de la roche. D'une manière générale, l'étude pétrographique de ces échantillons permet de mettre en évidence la grande ressemblance dans la nature minéralogique des deux parties du banc gréseux. Ainsi, la constitution minéralogique des deux lames est identique et rappelle en cela la composition minéralogique des niveaux sableux non consolidés dans les deux secteurs.

\section{Calcimétrie}

La teneur en carbonates dans ce banc varie de 5 à $25 \%$ au maximum. Le taux le plus élevé des carbonates s'enregistre dans les niveaux à texture fine. Ces carbonates sont d'origine secondaire, entraines par illuviation sous forme de particules argileuses ou en solution. 


\section{Relations stratigraphiques entre les dépôts du premier étage (Fig. 8)}

$\mathrm{Au}$ niveau du premier étage, le rapport stratigraphique se fait directement grâce à la formation sableuse qui constitue la couche 3 au niveau du secteur II du premier étage (profil 3) et la couche 2 des deux zones B et C $\mathrm{du}$ locus 6, profil 2. Ces sédiments d'origine allochtone ont des caractéristiques sédimentologiques identiques dans les deux secteurs. Ces caractéristiques se manifestent notamment au niveau de leur texture, structure, morphoscopie et minéralogie de leurs constituants. Cette formation sableuse est surmontée directement par les dépôts limoneux qui composent la couche 2 dans le secteur II, mais également la couche 1 dans la zone $\mathrm{C} \mathrm{du}$ locus 6 (premier étage).

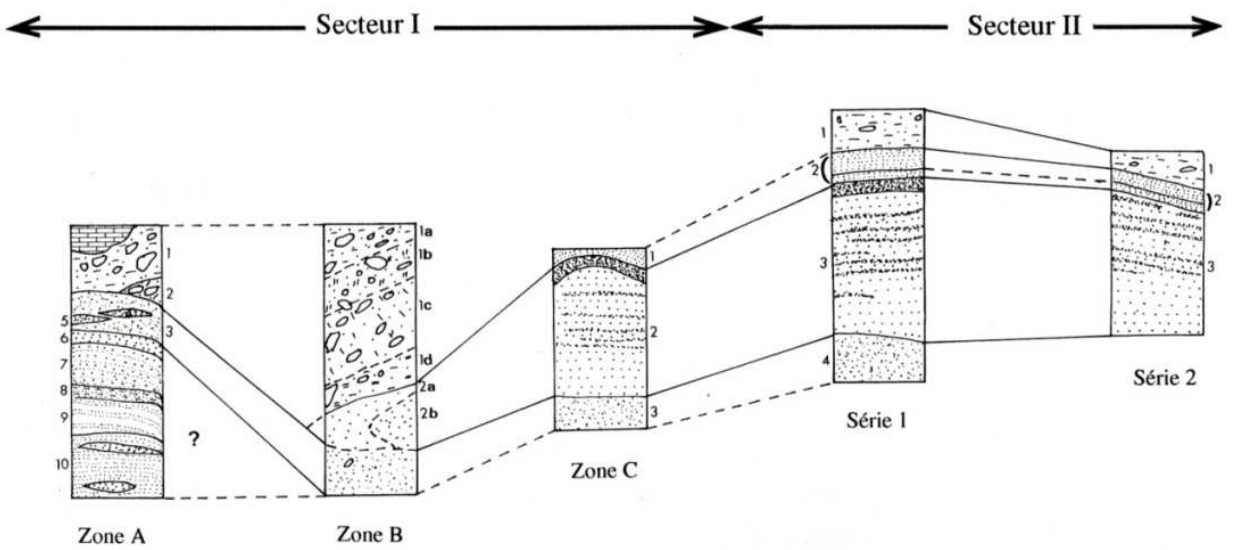

Fig. 8 : Relations stratigraphiques entre les différents secteurs et zones du premier étage

\section{Interprétations dynamique et climatique des dépôts}

Le dépôt de la couche 4 d'origine allochtone témoigne d'un climat tempéré et humide. Il a été transporté et mis en place par ruissellement diffus et de faible énergie. Ce dépôt a été également l'objet d'un important remaniement post sédimentaire.

En revanche, le caractère lité de la couche 3 montrent que cette dernière n'a été le fait d'aucun processus de remaniement. La sédimentation de ces matériaux s'est vraisemblablement déroulée pendant des épisodes tempérés et humides qui déterminent des ruissellements à régime plutôt soutenu susceptibles de transporter des sables grossiers, mais également des granules. Ces épisodes ont été suivis par des périodes assez chaudes et moins humides. Cette alternance a permis le développement des encroûtements par formation d'horizons d'accumulation des carbonates sous forme laminaire. Ces horizons sont plus ou moins continus au sein de cette formation sableuse et leur édification semble être influencée par l'effet de la paroi, ce qui explique les variations latérales de ce phénomène du concrétionnement. 
L'homogénéité de ces dépôts sableux indique une certaine régularité de l'intensité des ruissellements qui ont entrainés et mis en place ces sédiments. La proportion élevée des grains de quartz luisants et le bon classement des sables apportent un argument de plus à cette interprétation. Cependant, l'aspect "à peine mat" de certains grains peut avoir plusieurs explications. Parmi ces interprétations, on peut retenir l'héritage de ces matériaux à partir d'anciennes formations éolisées. D'autre part, on peut penser que cette catégorie de grains a été dépolie par altération chimique sous un climat assez chaud et humide (Tricart et Ali, 1958). Ce phénomène chimique peut être postérieur ou antérieur à leur mise en place. Toutefois, l'absence des grains de quartz typiquement éoliens (ronds mats) nous laisse envisager la deuxième hypothèse. Enfin, l'association de grains de quartz (sables et granules) à différents degrés d'usure, laisse penser à une reprise de sédiments plus ou moins anciens, sur des distances plus ou moins longues. La mise en place de ces dépôts d'origine allochtone à une dizaine de mètres au dessus du niveau actuel du cours d'eau de la Tardoire ne peut être expliquée que par une origine intra-karstique limitée dans l'espace, vu la situation topographique de cette formation à l'intérieur de la grotte. Le cas contraire de cette hypothèse pourrait provoquer la vidange des dépôts plus anciens à cette formation. L'inclinaison des niveaux sableux vers l'avant de la grotte apporte un argument supplémentaire à cette hypothèse. Il faut rappeler que le pendage de ces lits de sable perd progressivement de son importance au fur et à mesure qu'on s'approche du sommet de cette formation, et donc du comblement de cette partie du remplissage. Ce qui confirme la thèse de l'origine intrakarstique de ces dépôts.

La structure finement litée du dépôt limoneux qui représente les deux niveaux de la couche 2 (niveaux $2 \mathrm{a}$ et $2 \mathrm{~b}$ ) témoigne d'une mise en place de ces sédiments dans l'eau calme. Ces caractéristiques sédimentologiques et stratigraphiques montrent que ces dépôts ont été hérites d'anciens sédiments éolisés. Cependant, leur transport et leur mise en place correspondent à des conditions climatiques plutôt tempérées et humides, comme le confirme l'absence d'éléments carbonatés d'origine cryoclastique dans cette couche. La couche 1, constituée d'un sédiment assez fin, de quelques cailloutis et de blocs d'origine autochtone témoigne d'un climat relativement froid mais humide. Les ruissellements qui ont transportes et déposés une partie du sédiment fin non calcaire sont de faible compétence et diffus. La présence d'une proportion non négligeable de grains de quartz à aspect luisant ainsi que le taux assez important des argiles présentes dans ce dépôt confirment cette interprétation. 


\section{Conclusion}

Les études des sédiments proviennent du premier étage de la grotte de Montgaudier ont permis de conclure que les dépôts de cette partie du remplissage sont surtout d'origine allochtone. Ils ont été transportés et mis en place par ruissellement notamment au niveau des couches du secteur II où les sédiments qui les composent sont de nature siliceuses. L'aspect concrétionné d'une grande partie de ces dépôts explique le climat tempéré contemporain à l'édification de ces couches gréseuses. L'homogénéité des dépôts dans le secteur II indique une certaine régularité de l'intensité des ruissellements qui ont entrainés et mis en place ces sédiments. Les sédiments allochtones de ce remplissage sont en partie hérités des anciennes formations éoliennes comme en témoigne la présence de certains grains à aspect faiblement mat. Les structures finement litées des niveaux $2 \mathrm{a}$ et $2 \mathrm{~b}$ attestent d'une mis en place par un faible régime hydraulique. Ces analyses apportent des précisions qui sont chronologiquement fiables les unes par rapport aux autres. Enfin, une corrélation stratigraphique avec les dépôts adjacents au niveau d'abri Gaudry nous a apporté des arguments sur la période de cette partie du remplissage qui sont chronologiquement fiables par rapport aux dépôts d'abri Gaudry attribué au magdalénien (Duport 1971, 1981, Gambier D. 1986) ou en aurignacienne (Debenath 1974). En effet, et bien qu'aucune précision ne soit donnée sur cette datation, la corrélation stratigraphique établie grâce à ce travail, permet de placer cette partie du remplissage à la fin du Würm.

\section{References:}

1. BOUKHIR M. (2017). Etude sédimentologique et stratigraphique des dépôts des abris Gaudry et Lartet du gisement de Montgaudier, Charente, France. European Scientific Journal April 2017 edition Vol 11, No 2 (2015) pp. 249-266.

2. BOUKHIR M., LAHRACH A. ET CHAOUNI A. (2017). Etude minéralogique des fractions sableuse et argileuse du remplissage $\mathrm{du}$ gisement de Montgaudier, Charente, France. European Scientific Journal May 2017 edition Vol 13, No 15 (2017) pp. 195-215.

3. BOUKHIR M. (1992). Etude sédimentologique et stratigraphique du gisement de Montgaudier. Thèse de Doctorat d'Université, Bordeaux I. $543 p$.

4. DEBENATH A. (1974). Recherches sur les terrains quaternaires charentais et les industries qui leur sont associés. Doctorat d'état es sciences, université de Bordeaux I, 1974.

5. DUPORT L. (1971). Découverte de deux crânes humains dans la grotte de Montgaudier. C.R. Acad. Sc., Paris, 1971, 283: 1015-1016. 
6. DUPORT L. (1982). Le foyer magdalénien et la plaquette grave de Montgaudier, Charente. Bull. de la Sc. Arch. Et Hist. De la Charente, 1981-1982, pp : 23-30.

7. GAMBIER D. (1986). Les crânes magdaléniens de Montgaudier (Charente). In «préhistoire de Poitou-Charentes », problèmes actuels. Actes du 11ème congrès national des sociétés savantes, Poitiers 1986.

8. TRICART ET ALI (1958). Méthodes améliorée pour l'étude des sables. Revue de géomorphologie dynamique, Strasbourg, 1958, n) 3$4:$ 43-56.

9. VATAN A. Travaux pratiques de pétrographie sédimentaire (minéralogie). Editions TECHNIP, Paris, 1956. 